\title{
Epigenetic up-regulation of ribosome biogenesis and more aggressive phenotype triggered by the lack of the histone demethylase JHDM1B in mammary epithelial cells
}

\author{
Alice Galbiati ${ }^{1}$, Marianna Penzo ${ }^{1}$, Maria Giulia Bacalini ${ }^{1}$, Carmine Onofrillo ${ }^{1}$, Ania \\ Naila Guerrieri ${ }^{1}$, Paolo Garagnani ${ }^{1}$, Claudio Franceschi ${ }^{1}$, Davide Treré ${ }^{1}$, Lorenzo \\ Montanaro ${ }^{1}$ \\ ${ }^{1}$ Department of Experimental, Diagnostic, and Specialty Medicine, Alma Mater Studiorum, University of Bologna, Bologna, Italy \\ Correspondence to: Lorenzo Montanaro, email: Iorenzo.montanaro@unibo.it
}

Keywords: histone modification, JHDM1B, rDNA, ribosome biogenesis, cancer cells

Received: April 28, $2016 \quad$ Accepted: March 03, $2017 \quad$ Published: March 14, 2017

Copyright: Galbiati et al. This is an open-access article distributed under the terms of the Creative Commons Attribution License (CC-BY), which permits unrestricted use, distribution, and reproduction in any medium, provided the original author and source are credited.

\section{ABSTRACT}

The alterations of ribosome biogenesis and protein synthesis play a direct role in the development of tumors. The accessibility and transcription of ribosomal genes is controlled at several levels, with their epigenetic regulation being one of the most important. Here we explored the JmjC domain-containing histone demethylase $1 \mathrm{~B}$ (JHDM1B) function in the epigenetic control of rDNA transcription. Since JHDM1B is a negative regulator of gene transcription, we focused on the effects induced by JHDM1B knock-down (KD). We studied the consequences of stable inducible JHDM1B silencing in cell lines derived from transformed and untransformed mammary epithelial cells. In these cellular models, prolonged JHDM1B downregulation triggered a surge of 45S pre-rRNA transcription and processing, associated with a re-modulation of the H3K36me2 levels at rDNA loci and with changes in DNA methylation of specific CpG sites in rDNA genes. We also found that after JHDM1B KD, cells showed a higher ribosome content: which were engaged in mRNA translation. JHDM1B KD and the consequent stimulation of ribosomes biogenesis conferred more aggressive features to the tested cellular models, which acquired a greater clonogenic, staminal and invasive potential. Taken together, these data indicate that the reduction of JHDM1B leads to a more aggressive cellular phenotype in mammary gland cells, by virtue of its negative regulatory activity on ribosome biogenesis.

\section{INTRODUCTION}

Ribosome biogenesis, the process of ribosome production, is frequently up-regulated in cancer in order to respond to the increased demand of protein synthesis in highly proliferating cells. Indeed, this process is controlled by the same oncoproteins and tumor suppressors that normally control cell cycle progression, cell differentiation, and cell proliferation. Their de-regulation in tumors could be responsible for both a surge in ribosome biogenesis, and the loss of cell cycle control [1].

JmjC domain-containing histone demethylase 1B (JHDM1B, also called FBXL10 or KDM2B) is a conserved and ubiquitously expressed member of the JmjC domain-containing histone demethylase (JHDM) family involved in the demethylation of trimethylated lysine 4 on histone $\mathrm{H} 3$ (H3K4me3) and dimethylated lysine 36 on histone $\mathrm{H} 3$ (H3K36me2), thereby removing active chromatin marks and inhibiting gene transcription [2-4]. JmjC family members are able to specifically demethylate mono-, di-, and trimethylated lysines on the histone tails $[5,6]$. Great importance has recently been attributed to the negative regulatory function of JHDM1B through lysine H3K36me2 demethylation over a group of genes involved in senescence control, mapping at the Ink4a/Arf/Ink4b locus $[7,8]$. These genes encode for a series of critical cell cycle regulators: $\mathrm{p} 16^{\text {Ink4a }}$ and $\mathrm{p} 15^{\text {Ink4b }}$ prevent $\mathrm{G} 1 / \mathrm{S}$ phase transition through the inhibition of cyclin $\mathrm{D}$ binding to cyclin-dependent kinase 4 and 6 (Cdk4/6); p14 ${ }^{\text {Arf }}$ protein leads to cell cycle arrest or apoptosis, through p53 
pathway activation $[9,10]$. In this sense, JHDM1B acts as an oncoprotein that prevents proliferative senescence through Ink4a/Arf/Ink4b locus silencing [11]. Accordingly, it has been reported that this specific locus is frequently deleted or mutated in a variety of human primary tumors and that, in leukemia, JHDM1B overexpression drives tumorigenesis by overcoming cellular senescence $[12,13]$. On the other hand, a few but important observations involve JHDM1B in tumor suppression. In fact, it is reported to limit cell proliferation through the negative regulation of the transcription of the JUN protooncogene and rDNA genes [2, 14, 15]. Ribosomal genes are indeed present in hundreds of copies, but only a subset of the total pool is transcriptionally active at any given time due to their epigenetic inactivation [16]. In particular, the negative regulatory function of JHDM1B in ribosome biogenesis has been linked to $\mathrm{H} 3 \mathrm{~K} 4 \mathrm{me} 3$ demethylation at the rDNA level. In fact, the demethylase co-localizes with RNA polymerase I (PolI) and the Upstream Binding Factor (UBF) within Nucleolar Organizing Regions (NORs), preferentially binding the transcribed region of ribosomal DNA to repress its transcription [2]. In this sense, considering that low JHDM1B expression levels were found in aggressive brain and breast tumors characterized by an abnormally amplified ribosome biogenesis, we hypothesized that, despite its suppressive activity on proliferative senescence, the downregulation of this histone demethylase might contribute to tumor aggressiveness $[2,15]$. To verify the stated hypothesis, we investigated the role of JHDM1B-mediated negative control in ribosome biogenesis and its relevance to cancer, by focusing on the effects of JHDM1B downregulation on ribosome biogenesis and cell behavior in transformed and untransformed cells derived from mammary gland epithelium.

\section{RESULTS}

\section{JHDM1B downregulation variably affects the global histones marks}

The purpose of this work was to study the biological effects of long-lasting JHDM1B depletion on the transcription of ribosomal genes, as well as its contribution to the landscape of malignant phenotypes in mammary gland epithelial cells. To study the effects of long-lasting JHDM1B downregulation, we generated TRC-inducible shRNA cellular models starting from MDA-MB-231 and MCF 10A cells [termed hereafter MDA-MB-231 sh(1 or 2)-JHDM1B and MCF 10A sh(1 or 2)-JHDM1B]. After TRC treatment, we observed a drop in JHDM1B mRNA levels starting at $72 \mathrm{~h}$, which was recorded for at least 6 days in both MDA-MB-231 sh1-JHDM1B and MCF 10A sh1-JHDM1B cells (Figure 1A and 1B), thus indicating an early and stable effect of the shRNA expression. JHDM1B KD was associated with some changes in the global levels of the histone methylation set, measured by Western blot (Figure 1C and 1D). In particular, MDA-MB231-derived cells, after JHDM1B KD, showed increased global levels of histone $\mathrm{H} 3 \mathrm{~K} 4 \mathrm{me} 3$, while in the MCF $10 \mathrm{~A}$ the H3K36me2 histone mark increased after JHDM1B $\mathrm{KD}$, supporting a role played by this demethylase in the modification of these specific lysine residues. Nevertheless, in our conditions we did not observe significant changes in the mRNA expression of cyclin-dependent kinase inhibitor 2B (CDKN2B, p15), cyclin-dependent kinase inhibitor 1A genes (CDKN1A, p21), or polycomb silencing complex 1 (PRC1)-related genes, such as the enhancer of zeste homolog 2 (EZH2), and proto-oncogene polycomb ring finger (BMI1) (Supplementary Figure 1), even though these were previously shown to undergo a modulation after acute JHDM1B depletion $[15,17]$.

\section{JHDM1B downregulation stimulates 45S pre-rRNA transcription and processing}

Pulse-chase experiments aimed at evaluating the consequences of JHDM1B downregulation on rDNA transcription showed that JHDM1B KD affects the transcription of rDNA, by determining a conspicuous increase and a faster processing of neo-synthesized $45 \mathrm{~S}$ pre-rRNA (Figure 2A, 2C and Supplementary Figure 2). Accordingly, the incorporation of 5-fluoro uridine in nascent RNA was significantly increased after JHDM1B $\mathrm{KD}$ (Figure 2B and 2D), thus confirming an increase in rRNA transcription as a result of JHDM1B depletion. To rule out the possibility of off-target effects linked to the use of a single shRNA, we also achieved JHDM1B KD by expressing a different JHDM1B oligo in MDA-MB-231 and MCF 10A cells (MDA-MB-231 sh2-JHDM1B and $\mathrm{MCF}$ 10A sh2-JHDM1B); this demonstrated an increased $45 \mathrm{~S}$ pre-rRNA transcription during pulse-chase experiments also (Supplementary Figure 3).

\section{JHDM1B downregulation modifies H3K36 methylation at the rDNA level}

In order to investigate the role of JHDM1B in the modulation of histone markers at specific sites within ribosomal genes, we performed chromatin immunoprecipitation in MDA-MB-231 sh1-JHDM1B KD and MCF10A sh1-JHDM1B KD as well in their relevant controls, using an antibody raised against $\mathrm{H} 3 \mathrm{~K} 36 \mathrm{me} 2$, the major biochemical target of JHDM1B [3]. The purified chromatin was used in a quantitative PCR analysis in combination with six different pairs of primers mapping at the rDNA promoter, at four sequences located downstream of the start transcription site of the rDNA repeated units 4 (H4), 8 (H8), 13 (H13) and 30 (H30) kb and one mapping at the intergenic spacer region (IGS). The evaluation of the association of different $\mathrm{rDNA}$ regions and $\mathrm{H} 3 \mathrm{~K} 36 \mathrm{me} 2$ revealed a significant increase in the levels of this 
histone mark in $\mathrm{H} 4$ and $\mathrm{H} 8$ sequences after JHDM1B depletion in MDA-MB-231 cells (Figure 3A and 3B). No significant changes in $\mathrm{H} 3 \mathrm{~K} 36 \mathrm{me} 2$ composition were observed in the rDNA promoter region and at the H13, $\mathrm{H} 30$ and IGS regions. In MCF10A KD cells a significant increase of $\mathrm{H} 3 \mathrm{~K} 36 \mathrm{me} 2$ histone mark was observed in $\mathrm{H} 4$ and $\mathrm{H} 13$ regions, no particular changes in all the other analyzed regions (Figure 3C and 3D). Chromatin immunoprecipitation data obtained against total $\mathrm{H} 3$ histone as controls are also reported in (Supplementary Figure 4), showing that a global reorganization of histone $\mathrm{H} 3$ association with rDNA occurs after JHDM1B depletion in both cell lines, but with different behaviors.

These results suggest that JHDM1B may control chromatin status at the rDNA level, also acting on H3K36me2 .

\section{JHDM1B affects rDNA methylation}

Since JHDM1B histone demethylase can cause induce modifications in chromating status, it is conceivable that the methylation pattern of rDNA may also change

A

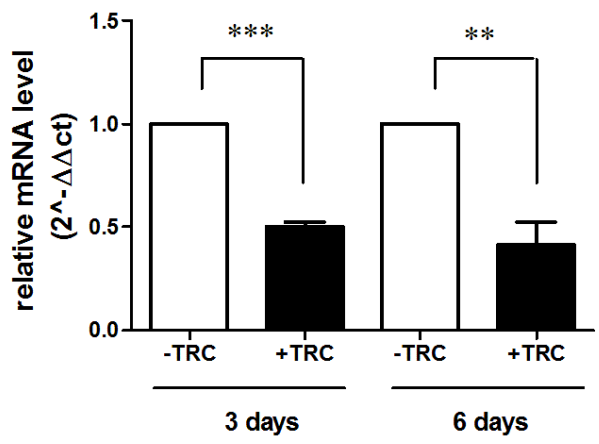

C

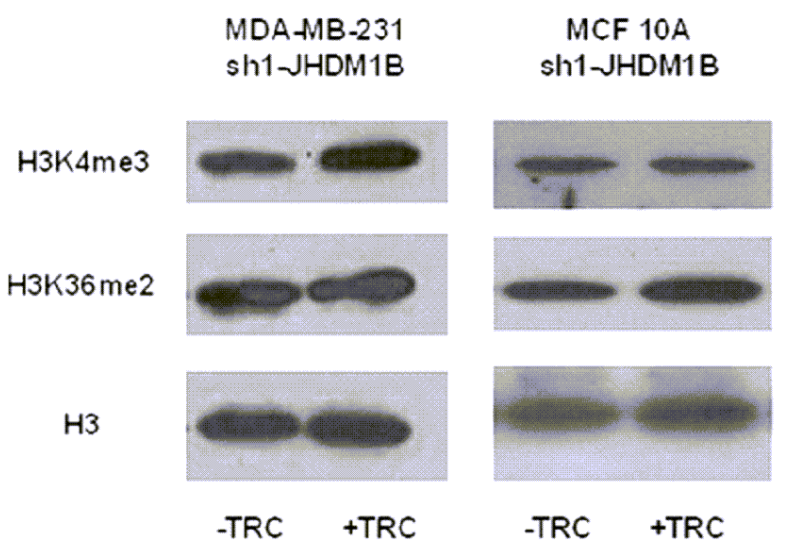

in JHDM1B KD cells. We used the EpiTYPER assay to quantitatively measure the methylation level of single CpG sites and small groups of adjacent CpGs (CpG units) in rDNA. In particular, we analyzed six target regions in ribosomal genes (Figure 4): a region $2 \mathrm{~kb}$ upstream of the upstream promoter of the ribosomal genes $(2 \mathrm{~kb}$ upstream), a region including both the upstream and the core promoters of ribosomal genes (RiboPromoter), the $5^{\prime}$ of $18 \mathrm{~S}$ sequence $(18 \mathrm{~S})$, the $5^{\prime}$ of $28 \mathrm{~S}$ sequence $(28 \mathrm{~S})$, and two regions overlapping with the H30 and the IGS sequences analyzed in chromatin immunoprecipitation experiments. Some of these regions partially overlap those previously analyzed in other studies [18]. After data cleaning we were able to measure the methylation levels of $10 \mathrm{CpG}$ units (containing $13 \mathrm{CpGs),} 8 \mathrm{CpG}$ units (containing $13 \mathrm{CpGs),} 14 \mathrm{CpG}$ units (containing 26 CpGs), $10 \mathrm{CpG}$ units (containing $15 \mathrm{CpGs),} 6 \mathrm{CpG}$ units (containing $6 \mathrm{CpGs)}$ and $13 \mathrm{CpG}$ units (14 CpGs) in $2 \mathrm{~kb}$ upstream, RiboPromoter, 18S, 28S, H30 and IGS target regions, respectively. In RiboPromoter amplicon, $7 \mathrm{CpGs}$ were localized in the upstream control element (UCE) region, while the remaining 6 were in the core
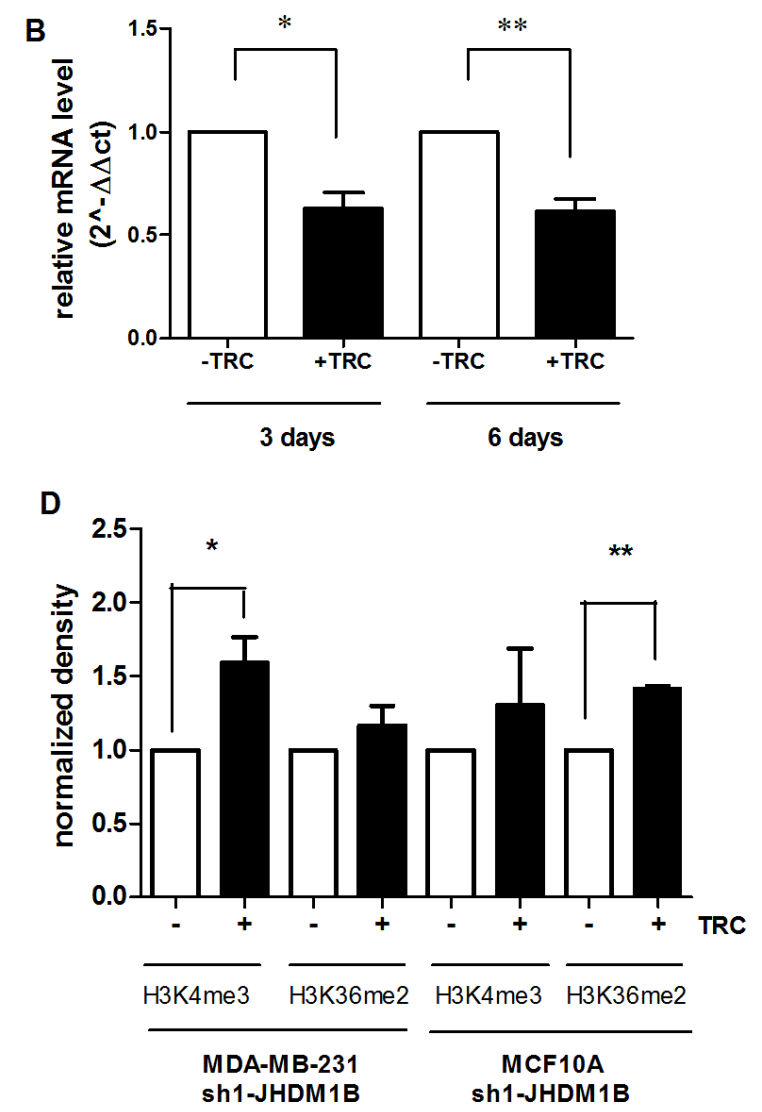

Figure 1: JHDM1B knock-down is associated with histone mark modification. (A and B) JHDM1B mRNA expression measured by real-time RT-PCR after 3 and 6 days of TRC administration in MDA-MB-231 sh1-JHDM1B (A) and MCF10 sh1-JHDM1B (B). Data were analyzed by paired Student's $T$-test: $* P<0.05$; ** $P<0.01$; *** $P<0.001$, (error bars, SEM). (C) Western blot analysis of the H3K4me3, H3K36me2 and total $\mathrm{H} 3$ histone levels in purified histones from MDA-MB-231 sh1-JHDM1B and MCF 10A sh1JHDM1B cells. (D) Densitometry of the gels shown in Figure 1C. The band densities corresponding to the H3K4me3 and H3K36me2 were normalized to those of the total $\mathrm{H} 3$ histone and subsequently data were normalized on the relative controls (-TRC). Results were analyzed by paired Student's $T$-test $* P<0.05,(N=3$, error bars, SEM). 
promoter. This analysis revealed that the methylation status of many $\mathrm{CpG}$ sites in rDNA changes after JHDM1B KD in MDA-MB-231 sh1-JHDM1B and MCF 10A sh1JHDM1B cells. In particular, in MDA-MB-231 sh1JHDM1B KD cells three $\mathrm{CpG}$ units were demethylated in the $18 \mathrm{~S}$ amplicon of MDA-MB-231 sh1-JHDM1B KD (18S_14.16; 18S_17.18, and 18S_35) and one was hypermethylated in the $\mathrm{H} 30$ amplicon (H30_10) (Figure 4A). In MCF 10A sh1-JHDM1B KD two CpG units of the $18 \mathrm{~S}$ were found to be demethylated (18S 2, 18S_28.29), and one unit of the same amplicon was hypermethylated (18S 35) after JHDM1B downregulation. The whole 28S amplicon appeared to be particularly affected by JHDM1B KD with many modified CpGs, including two sites (28S_2 and 28S_20), which were subjected to a significant demethylation (Figure 4B). Overall these results suggest that JHDM1B depletion induces a relaxation of rDNA chromatin at specific sites in the transcribed region thus allowing the access to DNA modifier enzymes. In the RiboPromoter, in the $2 \mathrm{~kb}$ upstream region and in the IGS region we were not able to find significantly different methylation patterns between control and silenced cells, suggesting that DNA methylation changes are locus-specific. The differences in methylation recorded at specific sites between the two cell lines may be ascribed to a different cell line-specific epigenetic asset.
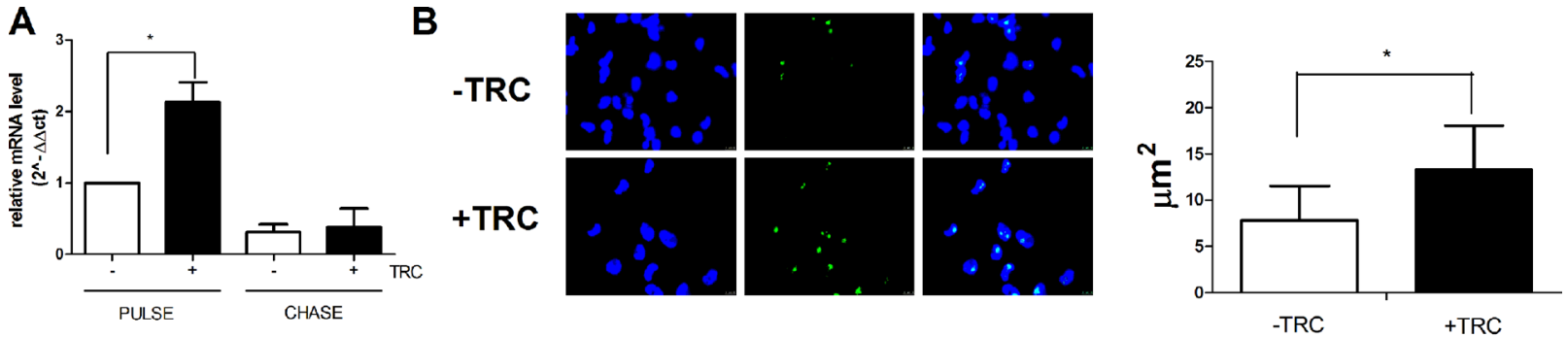

C

D

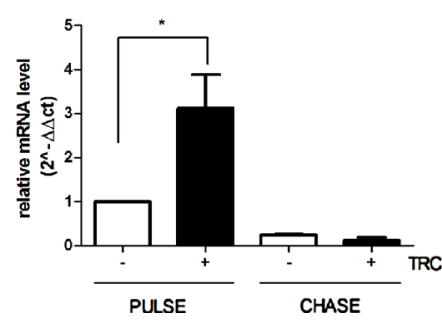

$-T R C$

+ TRC
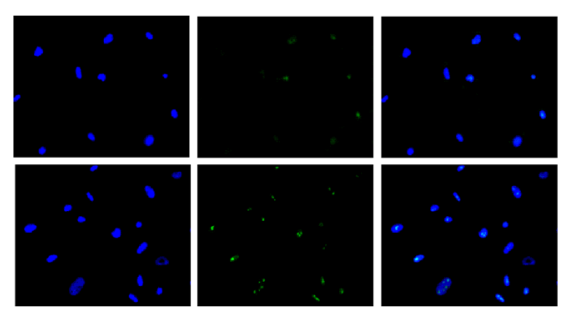

\section{Polysome profile analysis revealed an increased number of ribosomes involved in protein translation after JHDM1B KD}

In order to understand if the boosted transcription of $45 \mathrm{~S}$ pre-rRNA precursor was accompanied by an increase in ribosome production, we performed polysomal profile analysis via density fractioning of cellular lysates extracted from the same number of control and JHDM1B KD MDAMB-231 cells. This analysis revealed an increase in both the pre-polysomal and polysomal fractions absorbance in JHDM1B KD cells (Figure 5A); in addition, an enrichment of $18 \mathrm{~S}$ and $28 \mathrm{~S}$ rRNAs has been found in all the gradient fractions of the silenced MDA-MB-231 sh1-JHDM1B cells, particularly evident in the pooled polysomal fractions (Figure 5B), thus indicating that a higher number of ribosomes were involved in protein translation after JHDM1B reduction. Consistent results were observed when analyzing the amount of total and ribosomal RNA obtained from the same number of control and JHDM1B KD MCF10A cells (Supplementary Figure 5).

\section{JHDM1B downregulation triggers an aggressive behavior of mammary epithelial cells}

The inducible system we developed allows the analysis of prolonged JHDM1B depletion on different

Figure 2: JHDM1B knock-down causes a surge in $45 \mathrm{~S}$ pre-RNA synthesis and processing. (A) Evaluation of the neoproduced and processed 45S pre-rRNA in MDA-MB-231 sh1-JHDM1B. Cells were grown in medium supplemented with 5-ethynyl uridine for $1 \mathrm{~h}$ (pulse), or additionally grown in the presence of an excess of non-modified uridine for $2 \mathrm{~h}$ (chase), in order to evaluate respectively the neo-synthesized and processed $45 \mathrm{~S}$ pre-rRNA by real time RT-PCR. (B) Evaluation of the neo-produced and processed 45S pre-rRNA in MCF 10A sh1-JHDM1B. Cells were grown in medium supplemented with 5-ethynyl uridine for $2 \mathrm{~h}$ (pulse), or additionally grown in the presence of an excess of non-modified uridine for $2 \mathrm{~h}$ (chase), results were analyzed by paired Student's $T$-test; ${ }^{*} P<0.05$; $* * P<0.01$; $* * * P<0.001,(N=6$, error bars, SEM). (C) 5-fluoro uridine incorporation in nascent RNA in MDA-MB-231 sh1-JHDM1B (left) and average fluorescent nucleolar area (right). (D) 5-fluoro uridine incorporation in nascent RNA in MCF 10A sh1-JHDM1B (left) and average fluorescent nucleolar area (right). Dapi staining of nuclei (left blue signal), anti-mouse Alexa-488 (center green signal), merge of the two channels (right). Results were analyzed by unpaired Student's $T$-test $* P<0.05$ (error bars, SD). 
aspects of tumor cell behavior. We then explored various functional aspects such as growth and invasion capacity, ability to form colonies from individual cells, and mammosphere formation, in control and JHDM1Bdepleted MDA-MB-231 and MCF 10A cells. JHDM1B $\mathrm{KD}$ sustained over time caused an increase in MDAMB-231 sh1-JHDM1B cellular proliferation (Figure 6A). No effect on the growth behavior of MCF 10A sh1JHDM1B was observed (Figure 6B). JHDM1B KD led to a significantly enhanced colony-forming ability in both cell lines (Figure 6C and 6D). MDA-MB-231 and MCF 10A sh1-JHDM1B cells, as their relative controls, were still able to form mammospheres (MS) when grown on ultra-low attachment plates. MS can be formed from a niche of cells, with a staminal potential, able to grow as colonies in suspension in a syncytial state; in this condition the colony assumes a spherical shape [19, 20]. Both MDA-MB-231 and MCF 10A sh1-JHDM1B treated with TRC form a higher number of MS compared to control

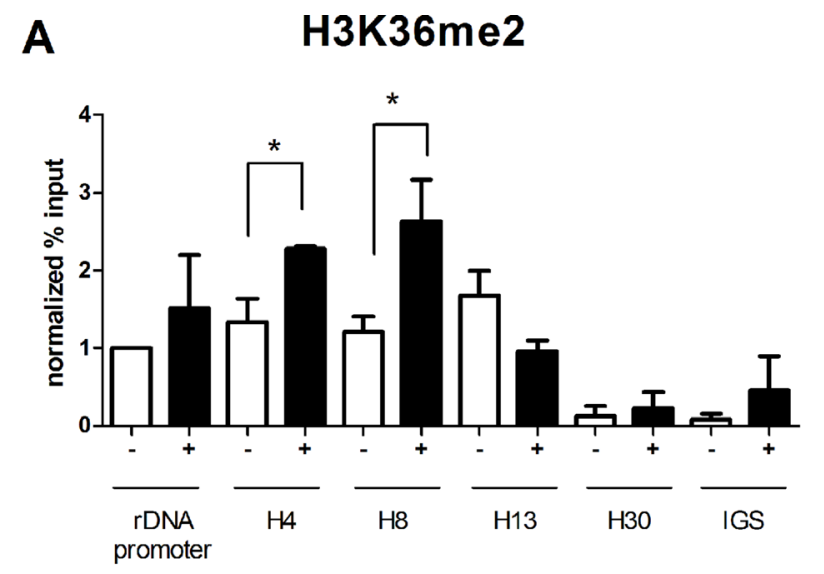

C

H3K36me2

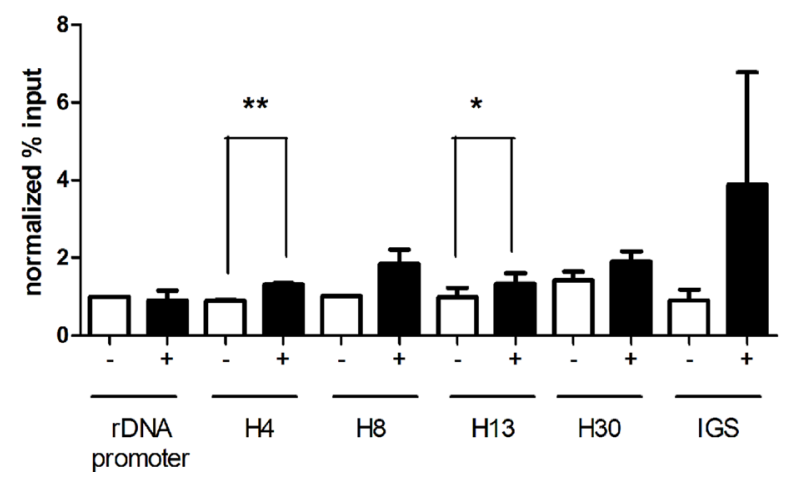

cells, indicating a greater staminal potential (Figure 6E and 6 F). Lastly, the invasion assays revealed a significant increase in the number of invading cells after JHDM1B $\mathrm{KD}$ in sh1-JHDM1B models (Figure $6 \mathrm{G}$ and $6 \mathrm{H}$ ). To rule out the possibility of off-target effects, we conducted invasion assays in MDA-MB-231 sh2-JHDM1B and MCF 10A sh2-JHDM1B, obtaining similar results (Supplementary Figure 6). Over all, these experiments indicate that the reduction of JHDM1B levels triggers an aggressive behavior of mammary gland epithelial cells, which is typical of cancer cells. Interestingly, MCF 10A sh1-JHDM1B, an untransformed cell line usually unable to form colonies and invade, acquired these skills after JHDM1B KD.

Since JHDM1B-depleted cells showed a more aggressive behavior, a MDA-MB-231 sh1-JHDM1B tetracycline-inducible shRNA model was used to generate tumors in nude Balb/c mice by injecting $2 \times 10^{6}$ cells into each of the animals' flanks (Supplementary Data).

B

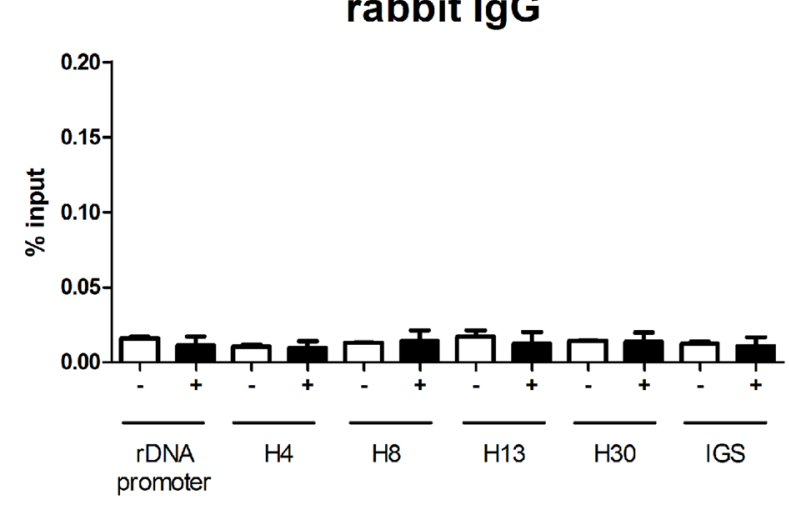

D

rabbit IgG

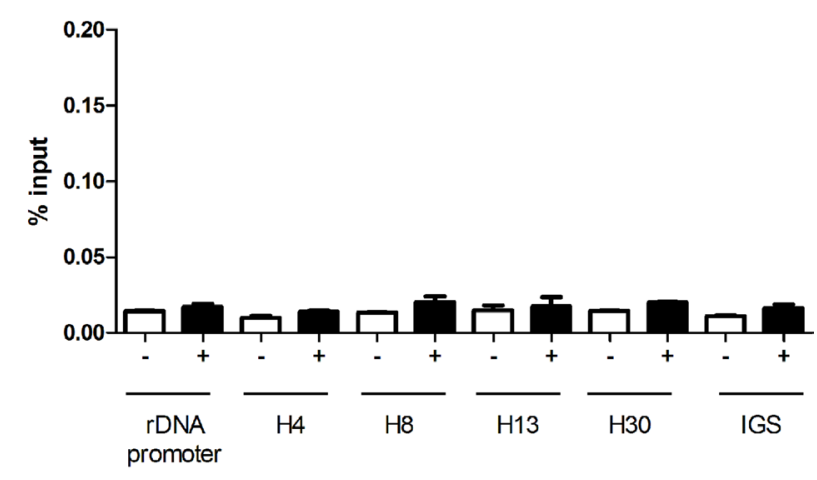

Figure 3: JHDM1B KD causes a modulation of the H3K36me2 mark at the rDNAlevel. (A) Chromatin immunoprecipitated with an anti-H3K36me2 in MDA-MB-231 sh1-JHDM1B control (white-filled) and KD cells (black-filled). (B) Chromatin immunoprecipitated with normal rabbit IgG in MDA-MB-231 sh1-JHDM1B control (white-filled) and KD cells (black-filled). (C) Chromatin immunoprecipitated with an anti-H3K36me2 in MCF10A sh1-JHDM1B control (white-filled) and KD cells (black-filled). (D) Chromatin immunoprecipitated with normal rabbit IgG in MCF10A sh1-JHDM1B control (white-filled) and KD cells (black-filled). Quantification obtained by real-time RT-PCR with specific primers for different regions within ribosomal genes: promoter, and three sequences located 4 (H4), 8 (H8), and 13 (H13) kb downstream of the start transcription site, one sequence located in the non transcribed spacer (H30) and one sequence located in the IGS region. Data were expressed as a $\%$ of the input without further normalization or normalized over the \% of input of rDNA promoter in control cells. The statistical analysis was by paired Student's $T$-test $* P<0.05 ; * * P<0.01(N=3$, error bars, SEM). 

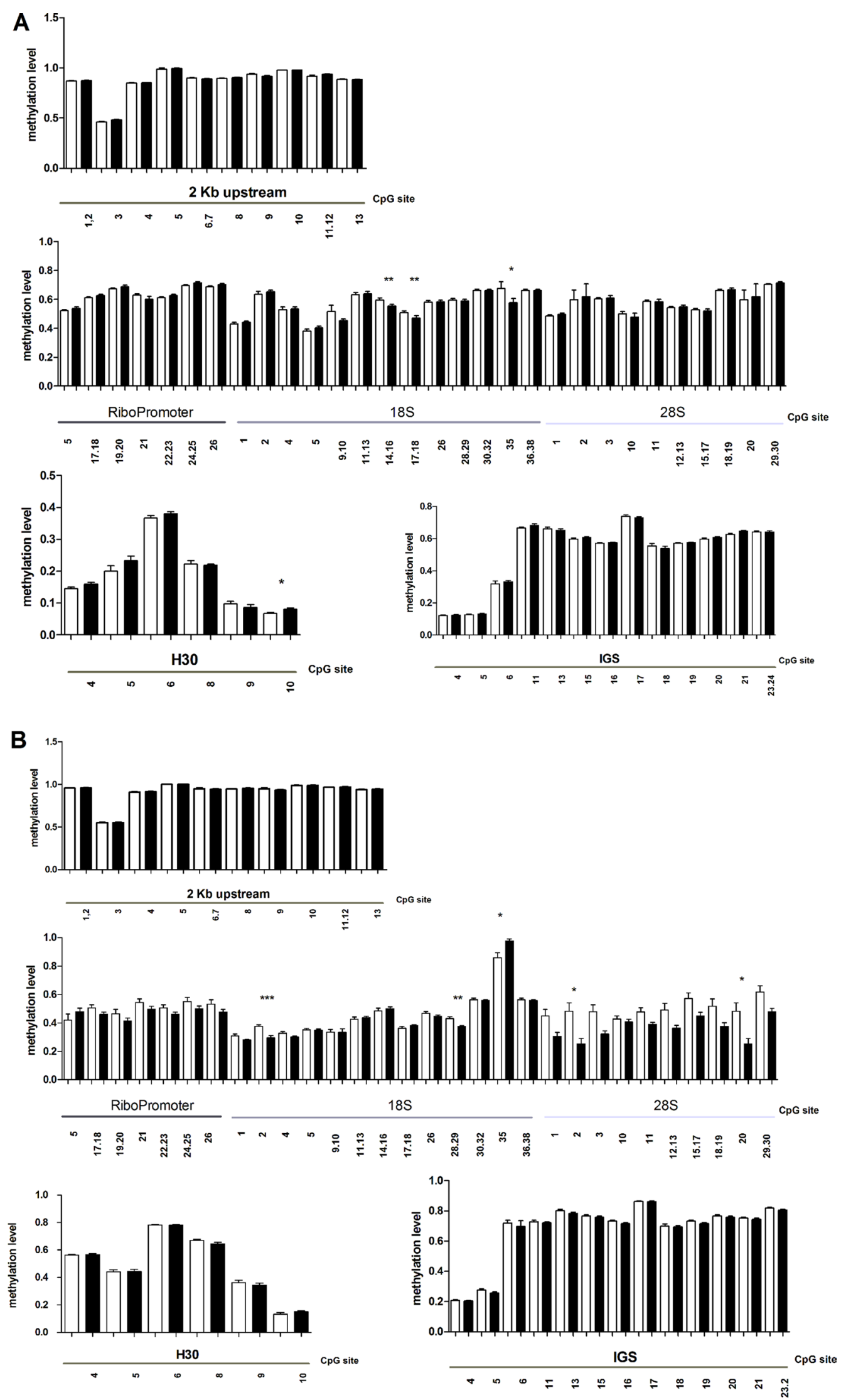

Figure 4: MassARRAY EpiTYPER analysis of KD (black-filled) and control cells (white-filled). (A and B) JHDM1B knock-down changes the methylation status of ribosomal genes: (A) MDA-MB-231 sh1-JHDM1B; (B) MCF 10A sh1-JHDM1B. Statistical analysis was by paired Student's $T$-test $* P<0.05 ; * * P<0.01 ; * * * P 0.001,(N=5$, error bars, SEM). 
After 9 weeks the mice were euthanized and the isolated tumors were measured. The obtained results indicated that JHDM1B KD triggered an incremental (although not statistically significant) trend in tumor growth (Supplementary Figure 7), while the silver nitrate staining of tumor sections (a technique widely used for selective nucleolar staining) revealed a different morphological conformation of the nucleoli after JHDM1B KD (Figure 7A). Tumors from TRC-treated mice showed a significant increase in the average nucleolar area compared to the untreated mice (Figure 7B), with a higher percentage of very large nucleoli $\left(>4 \mu \mathrm{m}^{2}\right)$ (Figure $7 \mathrm{C}$ ).

\section{DISCUSSION}

In this study we observed that the TRC-driven JHDM1B silencing in our cellular models caused a surge in $45 \mathrm{~S}$ pre-rRNA transcription, the rate-limiting step of ribosome biogenesis. The histone marks known to be the targets of JHDM1B were consequently modulated at different specific lysine residues looking to the global histone $\mathrm{H} 3$ methylation. In particular, the increased levels of H3K4me3 in MDA-MB-231 sh1-JHDM1B and higher level of H3K36me2 in MCF 10A sh1-JHDM1B were found after JHDM1B KD. On this concer, it should be considered that changes in the total levels of histone modifications after JHDM1B KD are not necessarily to be expected. Indeed, in the absence of global changes, it is still possible that focused lysine residue modifications occur at specific loci. Previous studies on Hela cells overexpressing JHDM1B mutated in its catalytic domain, demonstrated a decrease in the H3K4me3 level without affecting the global H3K36me2 mark [2] compared to the control cells expressing the wild type protein. In mouse embryo fibroblasts (MEF), no changes in the global levels of histone modification were found after JHDM1B overexpression, while in experiments of chromatin immunoprecipitation, a different $\mathrm{H} 3 \mathrm{~K} 4 \mathrm{me} 3$ and H3K36me2 composition was observed at specific loci [4]. To study histone mark changes at the rDNA level, we used chromatin immunoprecipitation and demonstrated for the first time that JHDM1B-downregulated cells increased the H3K36me2 level at the $4 \mathrm{~kb}$ and $8 \mathrm{~kb}$ transcribed regions of rDNA after KD, thereby proving that JHDM1B acts as a negative regulator of ribosome biogenesis not only by targeting the known $\mathrm{H} 3 \mathrm{~K} 4 \mathrm{me} 3$ residue, but also by acting on its major substrate $\mathrm{H} 3 \mathrm{~K} 36 \mathrm{me} 2$. This finding is strengthened by the recent observation that JHDM1A, a histone demethylase close to JHDM1B, acts on the same lysine residue in the negative regulation of rDNA transcription in response to glucose and serum starvation [21].

In addition, low levels of JHDM1B cause changes in rDNA methylation patterns, mainly resulting in a decreased level of methylation in the $18 \mathrm{~S}$ and $28 \mathrm{~S} \mathrm{CpG}$ islands, which falls in the rDNA regions known to be directly bound by JHDM1B [2]. The obtained general decrease in rDNA methylation is in line with a reduced chromatin condensation which is consequent to the lack of JHDM1B repressive function at the rDNA level. This observation is consistent with the increased rRNA transcription observed in JHDM1B KD. In the RiboPromoter region we were not
A

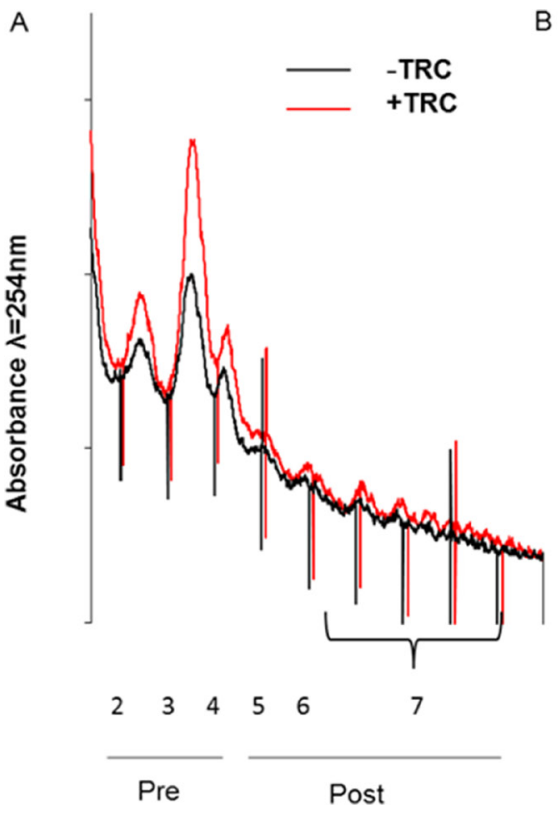

B

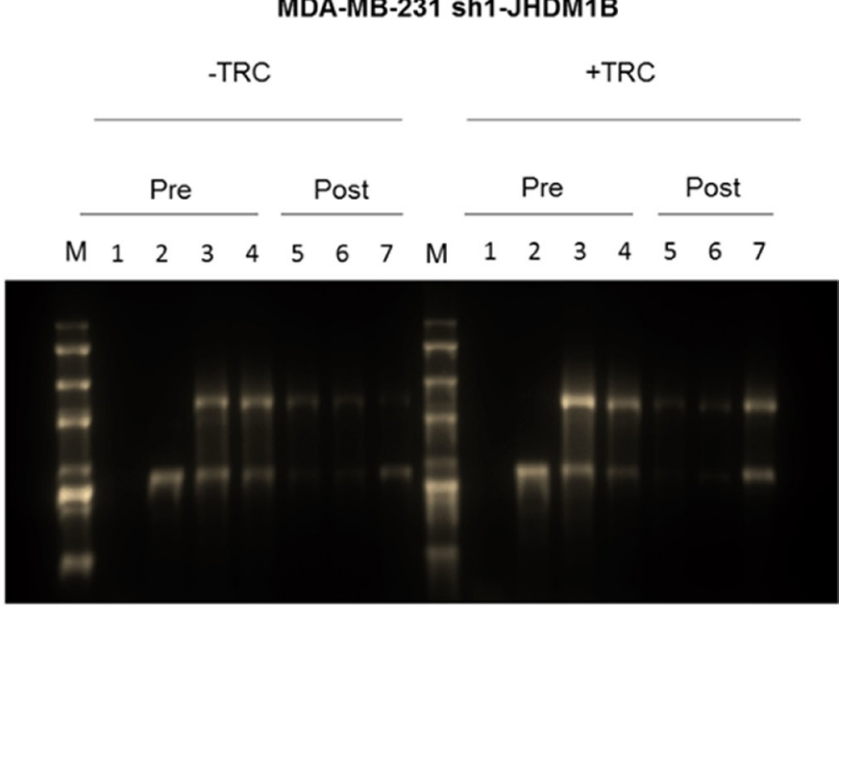

Figure 5: Polysome profile analysis of MDA-MB-231 sh1-JHDM1B cells. (A) Polysome profiles of control (black line) and $\mathrm{KD}$ cells (red line) obtained by measuring the absorbance at $254 \mathrm{~nm}$ in a continuous $15 \%-50 \%$ sucrose gradient. Numbers correspond to the sedimentation coefficients as follows: pre-polysomial 1) $<40 \mathrm{~S}$; 2) $40 \mathrm{~S}$; 3) $60 \mathrm{~S}$; 4) $80 \mathrm{~S}$, and post-polysomial > 80 5) disomes; 6) trisomes; and 7) the remaining polysome fractions. (B) Denaturing formaldehyde $1 \%$ agarose gel loaded with the total RNA extracted from each fraction obtained from the polysomal profile (M: $10000 \mathrm{bp}$ RNA marker). Polysome profiles and gel are representative images of 3 independent experiments. 

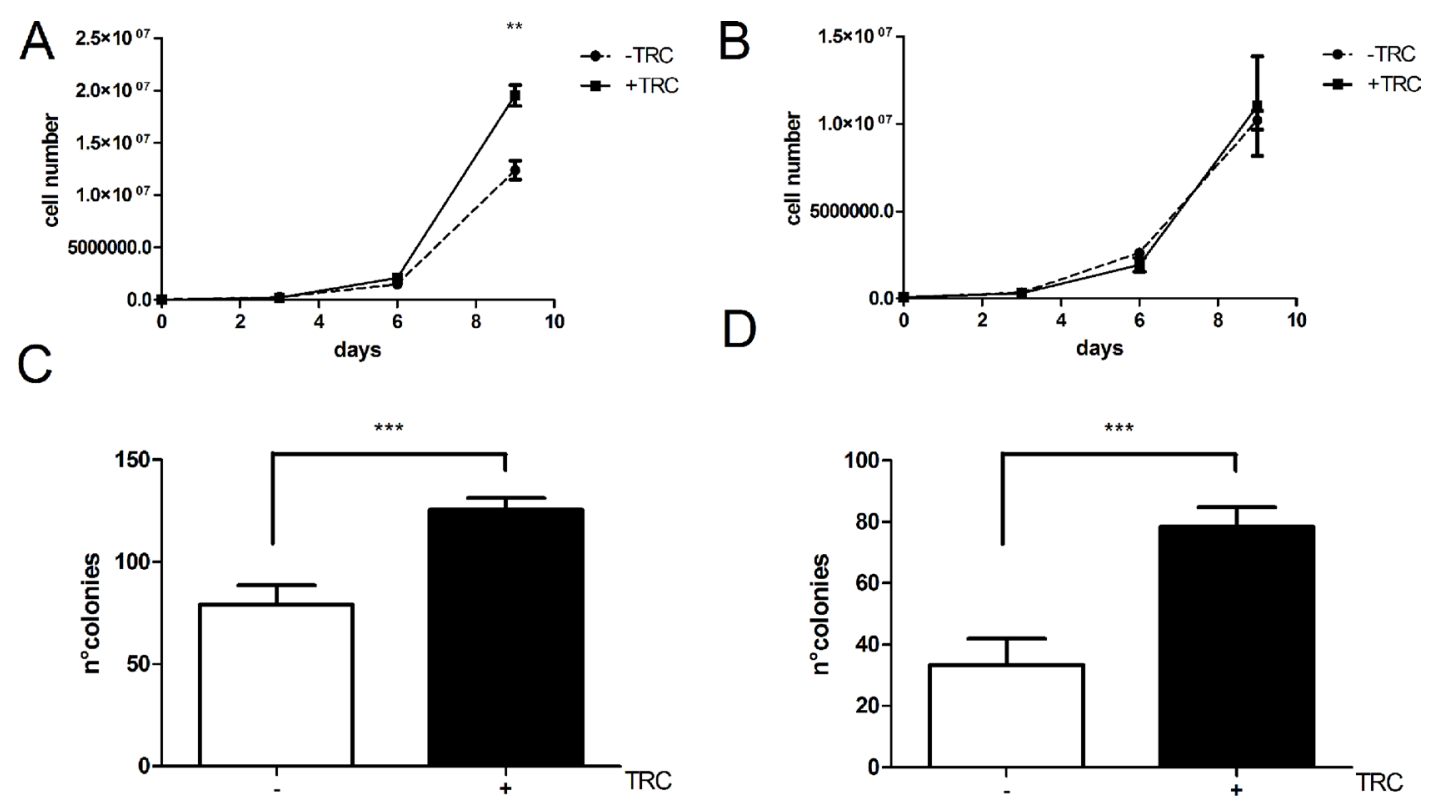

$E$

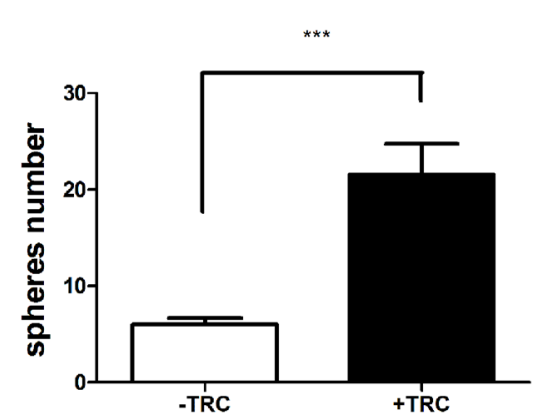

$\mathrm{F}$

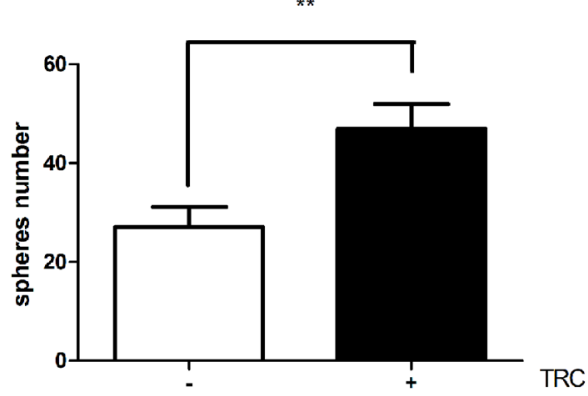

G

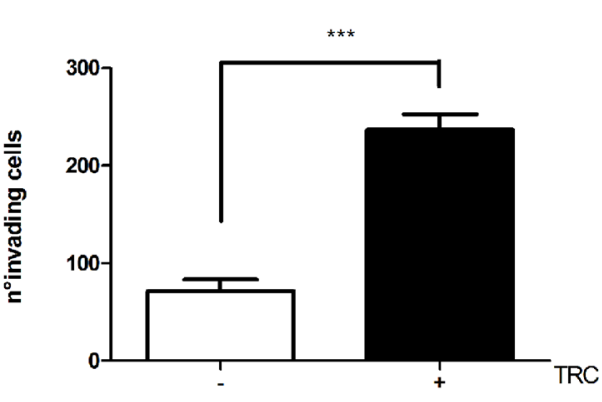

$\mathrm{H}$

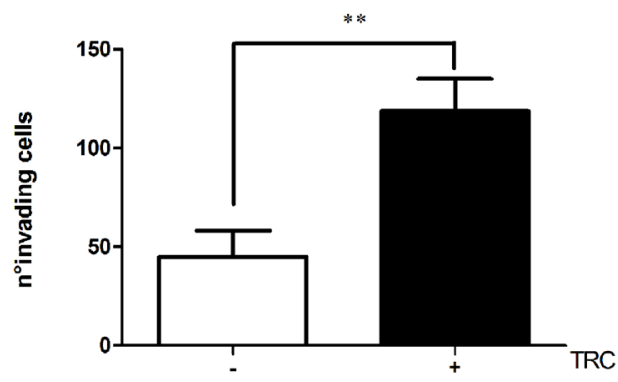

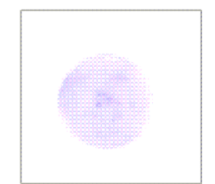

$-\mathrm{TRC}$

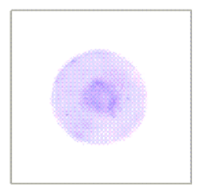

$+\mathrm{TRC}$

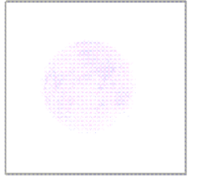

$-\mathrm{TRC}$

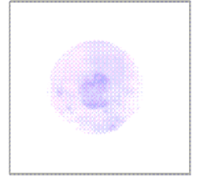

$+\mathrm{TRC}$

Figure 6: JHDM1B silencing triggers a more aggressive phenotype in the tested cell lines. (A) Growth curve profiles of control MDA-MB-231 sh1-JHDM1B cells (dotted line) and KD cells treated with TRC (solid line). (B) Growth curve profiles of control MCF 10A sh1-JHDM1B cells (dotted line) and KD cells (solid line) $(N=3$, error bars, SEM). (C and D) Colony numbers obtained from (C) MDA-MB-231 sh1-JHDM1B and (D) MCF 10 A sh1-JHDM1B ( $N=3$, error bars, SEM). (E and $\mathbf{F})$ Number of mammospheres produced by MDA-MB-231 sh1-JHDM1B (E) and MCF 10A sh1-JHDM1B (F). (G and H) Matrigel invasion assay of MDA-MB-231 sh1-JHDM1B (G) and MCF 10A sh1-JHDM1B (H). All graphs in the figure were obtained averaging at least three independent experiments (and no internal normalization between different experiments was performed), $p$ values were obtained by unpaired Student's $T$-test $* P<0.05$; $* * P<0.01$; $* * * P<0.001$ (error bars, SEM). The images below provide a representative picture of the matrigel-coated filters after $16 \mathrm{~h}$ of invasion. Photographs of 5 different areas were acquired for each filter, at $10 \times$ magnification, and used for cell counts. $(N=6$, error bars, SEM). 
able to find a significantly different methylation pattern between control and silenced cells. These results are in line with the available data that identify the $18 \mathrm{~S}$ and $28 \mathrm{~S}$ regions as primary binding sites for JHDM1B in rDNA, while a very low interaction is found between the demethylase and the promoter region [2].

JHDM1B KD did not represent a harmonic stimulus to the ribosome biogenesis, since in silenced cells the first effect is a boost in $45 \mathrm{~S}$ pre-rRNA production which is not expected to be paralleled by a concerted stimulation of all those factors which are important for a proper ribosome biogenesis. Despite this, we demonstrated that a higher number of ribosomes were present in cells with reduced JHDM1B levels and were involved in the protein synthesis process.

We also proved that the enhanced ribosome biogenesis conferred a growth advantage to MDAMB-231 cells, while this effect was not observe in MCF10A. Since these two cell lines display a different p53 status (wild type for MCF10A and mutated for MDAMB-231-[22], this observation is in line with our previous study showing that the effects of JHDM1B depletion on cellular proliferation are dependent on the status of p53 [15]. In addition, both tested cell lines acquired a greater clonogenic, staminal and invasive potential.
Taken together, these data support the role of JHDM1B as a tumor suppressor, since it is a negative regulator of ribosome biogenesis and cell proliferation. Apparently, the data obtained in our laboratory are in contrast with those reported by Kottakis et al., where JHDM1B silencing through a stable, and not inducible, lentiviral system in the same cell lines led to cell death by the activation of the Ink4a/Arf/Ink4b locus and PRC1 [17]. It is worthy of note that, in this study, a greater efficiency in gene silencing was obtained compared to that achieved with our inducible system, suggesting that the differences in the experimental approaches employed may be responsible for the different results observed

On the other hand, our data are consistent with those obtained after transient KD experiments in the same cellular models and with those that correlate JHDM1B mRNA levels in primary breast cancer specimens and patient clinical outcomes [15]. In particular, a worse prognosis was observed in cases in which the levels of the histone demethylase expression were below the median of the distribution, a situation comparable to the partial KD that we obtained in the present study.

In conclusion, the role of JHDM1B as an oncogene or tumor suppressor is still unclear and the subject of a widespread scientific discussion, but the data provided
A
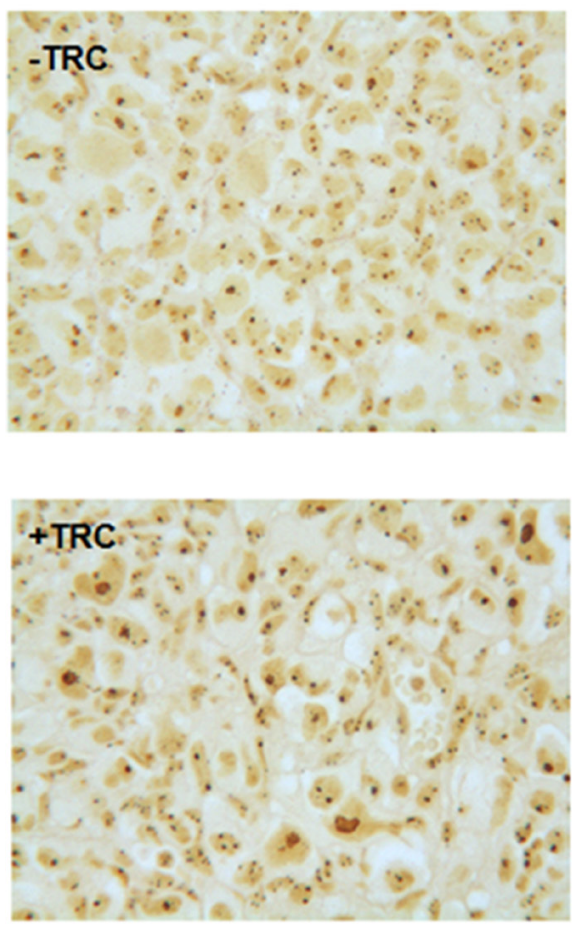

B

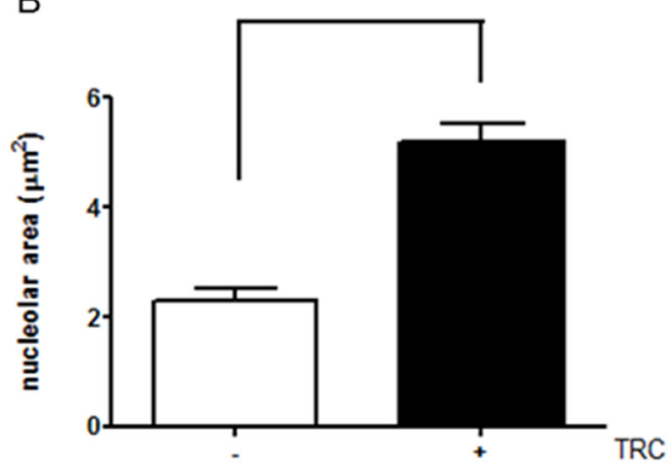

C

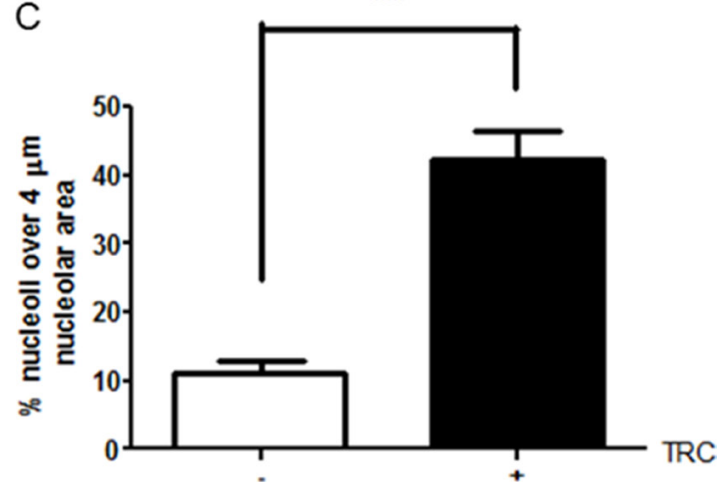

Figure 7: JHDM1B knock-down in MDA-MB-231 sh1-JHDM1B xenograft. (A) Representative images of AgNOR staining in MDA-MB-231 sh1-JHDM1B control tumor (upper panel) and TRC-treated mice (lower panel). Images show the presence of large nucleoli in JHDM1B KD xenograft. (B) Average nucleolar area measured in peripheral regions of 10 different tumors for each treatment. (C) Average percentage of large nucleoli (greater than $4 \mu \mathrm{m}$ ) in the total counted nucleoli. $p$ values were obtained using the unpaired Student's $T$-test $* * * P<0.001$ (error bars, SD). 
in this study support its role against breast cancer development and progression.

\section{MATERIALS AND METHODS}

\section{Cell culture and inducible shRNA interference}

MDA-MB-231 and MCF 10A cell lines were obtained from the American Type Culture Collection. During the study, cell line identity was verified by DNA fingerprinting and/or by day-by-day morphology checking. Cell lines expressing tetracycline inducible anti-JHDM1B shRNA, MDA-MB-231 sh(1 or 2)-JHDM1B, and MCF $10 \mathrm{~A} \operatorname{sh}(1$ or 2)-JHDM1B were generated by the BLOCKIT inducible H1 Lentiviral RNAi System following the manufacturer's instructions (Life Technologies). MDAMB-231 sh(1 or 2)-JHDM1B were cultured in DMEM supplemented with $10 \%$ Fetal Bovine Serum (FBS), $2 \mathrm{mM}$ L-Glutamine, $100 \mathrm{U} / \mathrm{ml}$ Penicillin and $1 \mathrm{mg} / \mathrm{ml}$ Streptomycin, $12 \mu \mathrm{g} / \mathrm{ml}$ Blasticidin, and $100 \mu \mathrm{g} / \mathrm{ml}$ Zeocin ( Sigma-Aldrich). MCF 10A sh(1 or 2)-JHDM1B were cultured in DMEM $1 \mathrm{~g} / \mathrm{L}$ glucose supplemented with $250 \mathrm{U} / \mathrm{L}$ of insulin, $0,5 \mu \mathrm{g} / \mathrm{ml}$ of hydrocortisone, $10 \mathrm{ng} / \mathrm{ml}$ of epidermal growth factor, 20\% (FBS), $2 \mathrm{mM} \mathrm{L}$-Glutamine, $100 \mathrm{U} / \mathrm{ml}$ Penicillin, and $1 \mathrm{mg} / \mathrm{ml}$ Streptomycin, $8 \mu \mathrm{g} / \mathrm{ml}$ of Blasticidin and $100 \mu \mathrm{g} / \mathrm{ml}$ Zeocin (Sigma-Aldrich). The expression of anti-JHDM1B shRNAs was obtained by the daily administration of Tetracycline (TRC) $1 \mu \mathrm{g} / \mathrm{ml}$ for sh1-JHDM1B cell lines, and $16 \mu \mathrm{g} / \mathrm{ml}$ for sh2JHDM1B. All experiments were carried out after 6 days of pre-induction of JHDM1B KD with TRC. To rule out the possibility that TRC treatment, rather than JHDM1B KD, affected cell proliferation, we investigated the effect of TRC administration on cell proliferation (Supplementary Figure 7A and 7B), and 5-fluoro uridine incorporation (Supplementary Figure 8C and 8D) in the parental cell lines MDA-MB-231 and MCF 10A. The double stranded DNA sequences used for the expression of the anti-JHDM1B shRNA were: sh1-JHDM1B forward: CACCGCAAACAGAGTGACATCTTTCCGAAGAAA GA; sh1-JHDM1B reverse: AAAAGCAAACAGAGTGA CATCTTTCTTCGGAAAGAT; sh2-JHDM1B forward: CACCGCATGAAGCAGAGCTGCATCACGAATGAT GC; sh2-JHDM1B reverse: AAAAGCATGAAGCAGAG CTGCATCATTCGTGATGC. All experiments were performed after the validation of the KD efficiency by real-time RT-PCR.Detailed protocols for cell proliferation, invasion, mammosphere production and colony formation assay were presented in the Supplementary Information (SI) section.

\section{RNA extraction and real-time RT-PCR}

RNAs were extracted using Tri-Reagent (Ambion), following the manufacturer's specifications. Real-time RT-PCR was performed as previously described [23]. Briefly, after RNA extraction and cDNA synthesis, a semi-quantitative Taqman approach (TaqMan Universal PCR master mix, Applied Biosystems) was used to evaluate the expression of JHDM1B, while a Sybr Green approach (Power SYBR green PCR master mix, Applied Biosystems) was used for the evaluation of $\beta$-actin as an endogenous control and $45 \mathrm{~S}$ pre-rRNA. Forward and reverse PCR primer sequences for Sybr green analysis of each mRNA were as follows: actin forward: ATCGTCCACCGCAAATGCTTCTA; actin reverse: AGCCATGCCAATCTCATCTTGTT; 45S pre-rRNA forward: GAACGGTGGTGTGTCGTTC; 45S pre-rRNA reverse: GCGTCTCGTCTCGTCTCACT.

\section{Histones extraction and western blot}

Cells were harvested and re-suspended in a TEB buffer $(0.5 \%$ Triton-X100, $2 \mathrm{mM}$ phenylmethyl sulfonylfluoride, $0,02 \% \mathrm{w} / \mathrm{v} \mathrm{NaN} 3$ in phosphate buffer) at a density of $1 \times 10^{7}$ cells $/ \mathrm{ml}$. Cells were then lysed on ice for $10 \mathrm{~min}$ and centrifuged at $10000 \mathrm{rpm}$ for $1 \mathrm{~min}$ at $4^{\circ} \mathrm{C}$; then the supernatant was removed. Cell pellets were suspended in 3 volumes of $10 \%$ glycerol, $0.5 \mathrm{~N} \mathrm{HCl}$, and incubated on ice for $30 \mathrm{~min}$. Samples were centrifuged at $12000 \mathrm{rpm}$ for $5 \mathrm{~min}$, and supernatants were placed into new tubes containing 8 volumes of acetone for overnight precipitation at $-20^{\circ} \mathrm{C}$. Samples were centrifuged at $12000 \mathrm{rpm} 20 \mathrm{~min}$; then supernatants were removed and the dried pellets were resuspended in distilled water. Histones were quantified with Bradford staining (BioRad) following the standard protocol. $5 \mu \mathrm{g}$ of total histones were loaded on $10 \%$ acrylamide gel to be evaluated by Western blotting histone H3 (Abcam, ab1791), H3K4me3 (Abcam, ab8580), and H3K36me2 (Abcam, ab9049).

\section{S pre-rRNA transcription and processing evaluation}

The level of newly synthesized 45S pre-rRNA was evaluated using the Click-iT Nascent RNA Capture Kit following the manufacturer's specifications (Life Technologies). $3 \times 10^{5}$ cells were seeded in a $35 \mathrm{~mm}$ dish the day before the experiments. Cells were treated with $0.1 \mathrm{mM} 5$-ethynil uridine in culture medium for $1 \mathrm{~h}$ or $2 \mathrm{~h}$ (pulse), respectively, for MDA-MB-231 sh(1 or 2)-JHDM1B and MCF 10A sh(1 or 2)-JHDM1B. For the evaluation of $45 \mathrm{~S}$ pre-rRNA processing, cells were cultured for 2 additional hours in the presence of an unmodified uridine excess at a concentration of $0.2 \mathrm{mM}$ (chase).

\section{Immunofluorescent detection of rRNA synthesis}

Immunodetection of the nascent rRNA was performed by incorporating 5-fluoro uridine, according to a method described elsewhere [24]. Samples were observed under a Leica DMI4000B inverted fluorescence microscope (Leica Microsystems, Milan, Italy) and analyzed using Image-Pro Analyzer Software. 


\section{Chromatin immunoprecipitations of H3K36me2}

Chromatin immunoprecipitations were performed on MDA-MB-231 sh1-JHDM1B and MCF10A sh1-JHDM1B cells using the EZ-Chip Kit following the manufacturer's instructions (Merk-Millipore). In particular, immunoprecipitations were performed by incubating the precleared chromatin, diluted $1: 15$, overnight at $4^{\circ} \mathrm{C}$ with $10 \mu \mathrm{g}$ of specific primary antibodies [rabbit antiH3K36me2 (Abcam, ab9049), rabbit anti-H3 (Abcam, ab1791) and normal rabbit IgG (Millipore, 12-370)], while $10 \mu \mathrm{l}(1 \%)$ of each sample were set aside as "input". The immunoprecipitated DNA was extracted with a QIAquick PCR purification kit (Qiagen) and used for quantitative RT-PCR analysis. Primer sequences used for H4, H8, and H13 regions have been published elsewhere [25]. Primer sequences for other rDNA regions were: rDNA promoter forward: GGTATATCTTTCGCTCCGAG; rDNA promoter reverse: AGCGACAGGTCGCCAGAGGA. H30 forward: ACTGGCGAGTTGATTTCTGG; H30 reverse: CGAGACAGTCGAGGGAGAAG; IGS forward: CACTACCCACGTCCCTTCAC; IGS reverse: GAGAGAAGACGGAGGCACAC.

\section{EpiTYPER assay for the quantitative DNA methylation analysis}

Quantitative methylation analysis of rDNA locus was performed using the EpiTYPER assay (Sequenom). Briefly, 1000 ng of DNA were bisulfite-converted using the EZ-96 DNA Methylation Kit (Zymo Research Corporation) as previously described [26]. $10 \mathrm{ng}$ of bisulfite-treated DNA were PCR-amplified using already validated primer pairs, three of which were previously analyzed using the same technique [27]: i) $2 \mathrm{~kb}$ upstream, from position -2274 to position -2055 (with respect to the transcription start site), forward primer AGGAAGAGA GGGGAGTTGGAGATTAGTTTGAGTAA, reverse primer CAGTAATACGACTCACTATAGGGAGAAGGC TTAAAACAAAATTTCACTCTTATTTCCAC; ii) RiboPromoter, from position -186 to position +48 , including both the upstream and core promoters of the ribosomal gene [27]; iii) $18 \mathrm{~S}$, from position +2946 to position +3432 , encompassing the 5 -sequence of the $18 \mathrm{~S}$ region [27]; iv) $28 \mathrm{~S}$, from position +7297 to position +7579 , encompassing the $5^{\prime}$-sequence of the $28 \mathrm{~S}$ region [27]; v) the H30, forward primer AGGAAGAGAGAGGA TTTTGGGTAGGAAAGTTTTT, reverse primer CAGT AATACGACTCACTATAGGGAGAAGGCTCCCAAATT TAATCTCCCTACAAAATA; vi) the IGS, forward primer AGGAAGAGAGAGGGGGGTTTTATTGTTTTTTGA, reverse primer CAGTAATACGACTCACTATAGGGAG AAGGCTAAAAAAAACCTCACAACTACAAACC.

For each target region, the assay returns the methylation level (expressed as a continuous number ranging from 0 to 1) of either single $\mathrm{CpG}$ sites or small groups of adjacent $\mathrm{CpG}$ sites. The quality control of EpiTYPER results was performed to remove $\mathrm{CpG}$ sites/units with missing values in more than $20 \%$ of the samples, as well as samples with missing values in more than $20 \%$ of $\mathrm{CpG}$ sites/units.

\section{Polysome profile analysis}

A separation based on the sedimentation coefficients of active translation machinery was performed in $15 \%$ to $50 \%$ continuous sucrose gradient in LSB $1 \mathrm{X}+$ Cycloheximide (CHX) [20 mM Trizma Base pH 7.5, $10 \mathrm{mM} \mathrm{NaCl}, 3 \mathrm{mM}$ $\mathrm{MgCl}_{2}, 100 \mu \mathrm{g} / \mathrm{ml} \mathrm{CHX}$ and $0.04 \mathrm{U} / \mu \mathrm{l}$ RiboLock RNAse inhibitor (Thermo Scientific)]. $5 \times 10^{6}$ MDA-MB-231 sh1JHDM1B KD and control cells were seeded in a $150 \mathrm{~mm}$ cell culture dish. After 24 hours, cells were treated with CHX $(100 \mu \mathrm{g} / \mathrm{ml})$ for $20 \mathrm{~min}$ at $37^{\circ} \mathrm{C}, 5 \% \mathrm{CO}_{2}$, washed twice with PBS + CHX $(100 \mu \mathrm{g} / \mathrm{ml})$, and harvested by scraping. The entire procedure was performed on ice and in the presence of CHX for RNA-ribosome complex maintenance. Cell pellets were re-suspended in $150 \mu \mathrm{l}$ of LSB 1X supplemented with $0.3 \%$ Triton N-101, $50 \mathrm{mM}$ sucrose, $100 \mu \mathrm{g} / \mathrm{ml}$ CHX and $0.04 \mathrm{U} / \mu \mathrm{l}$ RiboLock RNase inhibitor and protease inhibitor cocktail (Roche), and incubated for $10 \mathrm{~min}$ on ice. Total proteins were quantified by Bradford assay before loading lysates on gradients. Lysates were cleared by centrifugation at $14000 \mathrm{~g}$ for $10 \mathrm{~min}$ at $4^{\circ} \mathrm{C}$. Ribosomes were then separated on the continuous sucrose gradient by ultracentrifugation at $160000 \mathrm{~g}$ for $2 \mathrm{~h}$ at $4^{\circ} \mathrm{C}$. Polysome profile was monitored at $254 \mathrm{~nm}$ ( 0.2 OD sensitivity) and fractionated (at 10×, 10\% TRIS-Pump power) using an ISCO gradient fractionator system interfaced to an UA-6 absorbance detector (Teledyne Isco, Lincoln, NE, USA). Collected data were digitally converted by using Minilab 100 (Measuring Computing, Norton, MA, USA) and TracedDaq software (Measuring Computing, Norton, MA, USA), by acquiring data in differential mode at $+/-4 \mathrm{~V}$ and $4 \mathrm{~Hz}$.

RNA extraction by polysomal fractions and electrophoresis were discussed in the Supplementary Information section (SI).

\section{Selective nucleolar staining}

Five-micron sections were processed and silverstained to visualize nucleolar organizer regions and argyrophilic proteins according to the guidelines of the "International Committee on AgNOR Quantitation" [28]. Silver-stained sections were examined with a Leitz Diaplan light microscope (Wetzlar Germany) equipped with a video camera (JVC, 3CCD, KY-F55B, Jokohama, Japan), and analyzed using Image-Pro Analyzer Software.

\section{Statistical analysis}

Appropriate statistical analyses were performed as indicated in the figure legends, using the paired or unpaired Student's $T$-test. Data analyses were performed by GraphPad Prism 5.0 and Microsoft Excel. 


\section{ACKNOWLEDGMENTS}

The authors wish to thank the Center for Applied Biomedical Research (CRBA) of S. Orsola-Malpighi University Hospital for providing a number of instruments. They are also grateful to Dr. Massimiliano Bonafè and Sabrina De Carolis of the Department of Experimental, Diagnostic and Specialty Medicine, University of Bologna, S. Orsola-Malpighi University Hospital for their support in the mammosphere experiments.

\section{CONFLICTS OF INTEREST}

The authors declare no conflicts of interest.

\section{GRANT SUPPORT}

Associazione Italiana per la Ricerca sul Cancro (Italian Association for Cancer Research: AIRC); Grant number: IG-11416 to L.M. Italian Ministry of Education, University and Research PRIN Grant number: 20104AE23N 002 to L.M.; Pallotti legacy for Cancer Research to D.T. and L.M.; M.P. is a fellow of Fondazione Umberto Veronesi.

\section{REFERENCES}

1. Montanaro L, Trerè D, Derenzini M. Changes in ribosome biogenesis may induce cancer by down-regulating the cell tumor suppressor potential. Biochim Biophys Acta. 2012; 1825:101-110.

2. Frescas D, Guardacivaccaro D, Bassermann F, KoyamaNasu R, Pagano M. JHDM1B/FBXL10 is a nucleolar protein that represses transcription of ribosomal RNA genes. Nature. 2007; 450:309-313.

3. Nakamura S, Tan L, Nagata Y, Takemura T, Asahina A, Yokota D, Yagyu T, Shibata K, Fujisawa S, Ohnishi K. JmjC-domain containing histone demethylase 1B-mediated p15(Ink4b) suppression promotes the proliferation of leukemic progenitor cells through modulation of cell cycle progression in acute myeloid leukemia. Mol Carcinog. 2013; 52:57-69.

4. Janzer A, Stamm K, Becker A, Zimmer A, Buettner R, Kirfel J. The H3K4me3 histone demethylase Fbxl10 is a regulator of chemokine expression, cellular morphology, and the metabolome of fibroblasts. J Biol Chem. 2012; 287:30984-30992.

5. Mosammaparast N, Shi Y. Reversal of histone methylation:biochemical and molecular mechanisms of histone demethylases. Annu Rev Biochem. 2010; 79:155-179.

6. Lee JH, Voo KS, Skalnik DG. Identification and characterization of the DNA binding domain of CpGbinding protein. J Biol Chem. 2001; 276:44669-44676.
7. Popov N, Gil J. Epigenetic regulation of the INK4b-ARFINK4a locus in sickness and in health. Epigenetics. 2010; $5: 685-690$.

8. Tzatsos A, Pfau R, Kampranis SC, Tsichlis PN. Ndy1/ KDM2B immortalizes mouse embryonic fibroblasts by repressing the Ink4a/Arf locus. PNAS. 2009; 106:2641-2646.

9. Gil J, Peters G. Regulation of the INK4b-ARF-INK4a tumour suppressor locus:all for one or one for all. Nat Rev Mol Cell Biol. 2006; 7:667-677.

10. Ortega S, Malumbres M, Barbacid M. Cyclin D-dependent kinases, INK4 inhibitors and cancer. Biochim Biophys Acta. 2002; 1602:73-87.

11. He J, Kallin EM, Tsukada Y, Zhang Y. The H3K36 demethylase $\mathrm{Jhdm} 1 \mathrm{~b} / \mathrm{Kdm} 2 \mathrm{~b}$ regulates cell proliferation and senescence through p15Ink4b. Nat Struct Mol Biol. 2008; 15:1169-1175.

12. Okuda T, Shurtleff SA, Valentine MB, Raimondi SC, Head DR, Behm F, Curcio-Brint AM, Liu Q, Pui CH, Sherr CJ, Beach D, Look AT, Downing JR. Frequent deletion of p16INK4a/MTS1 and p15INK4b/MTS2 in pediatric acute lymphoblastic leukemia. Blood. 1995; 85:2321-2330.

13. Ogawa S, Hirano N, Sato N, Takahashi T, Hangaishi A, Tanaka K, Kurokawa M, Tanaka T, Mitani K, Yazaki Y. Homozygous loss of the cyclin-dependent kinase 4-inhibitor (p16) gene in human leukemias. Blood. 1994; 84:2431-2435.

14. Koyama-Nasu R, David G, Tanese N. The F-box protein Fbl10 is a novel transcriptional repressor of c-Jun. Nat Cell Biol. 2007; 9:1074-1080.

15. Penzo M, Casoli L, Pollutri D, Sicuro L, Ceccarelli C, Santini D, Taffurelli M, Govoni M, Brina D, Trerè D, Montanaro L. JHDM1B expression regulates ribosome biogenesis and cancer cell growth in a p53 dependent manner. Int J Cancer. 2015; 136:272-281.

16. Espada J, Ballestar E, Santoro R, Fraga MF, Villar-Garea A, Németh A, Lopez-Serra L, Ropero S, Aranda A, Orozco H, Moreno V, Juarranz A, Stockert JC, et al. Epigenetic disruption of ribosomal RNA genes and nucleolar architecture in DNA methyltransferase 1 (Dnmt1) deficient cells. Nucleic Acids Res. 2007; 35:2191-2198.

17. Kottakis F, Foltopoulou P, Sanidas I, Keller P, Wronski A, Dake BT, Ezell SA, Shen Z, Naber SP, Hinds PW, McNiel E, Kuperwasser C, Tsichlis PN. NDY1/KDM2B functions as a master regulator of polycomb complexes and controls self-renewal of breast cancer stem cells. Cancer Res. 2014; 74:3935-3946.

18. Javierre BM, Fernandez AF, Richter J, Al-Shahrour F, Martin-Subero JI, Rodriguez-Ubreva J, Berdasco M, Frega MF, O'Hanlon TP, Rider LG, Jacinto FV, LopezLongo FJ, Dopazo J, et al. Changes in the pattern of DNA methylation associate with twin discordance in systemic lupus erythematosus. Genome Res. 2010; 20:170-179. 
19. Veneziani BM, De Placido S. Issues of banking breast cancer cells to generate mammospheres. Cell Tissue Bank. 2013; 14:153-158.

20. Papi A, Guarnieri T, Storci G, Santini D, Ceccarelli C, Taffurelli M, De Carolis S, Avenia N, Sanquinetti A, Sidoni A, Orlandi M, Bonafé M. Nuclear receptors agonists exert opposing effects on the inflammation dependent survival of breast cancer stem cells. Cell Death and Differentiation. 2012; 19:1208-1219.

21. Tanaka Y, Yano H, Ogasawara S, Yoshioka SI, Imamura H, Okamoto K, Tsuneoka M. Mild glucose starvation induces KDM2A-mediated H3K36me2 demethylation through AMPK to reduce rRNA transcription and cell proliferation. Mol Cell.Biol. 2015; 35:4170-4184.

22. Kao J, Salari K, Bocanegra M, Choi YL, Girard L, Gandhi J, Kwei KA, Hernandez-Boussard T, Wang P, Gazdar AF, Minna JD, Pollack JR. Molecular profiling of breast cancer cell lines defines relevant tumor models and provides a resource for cancer gene discovery. PLoS One. 2009; 4:e6146.

23. Montanaro L, Calienni M, Bertoni S, Rocchi L, Sansone P, Storci G, Santini D, Ceccarelli C, Taffurelli M, Carnicelli D, Brigotti M, Bonafè $\mathrm{M}$, Treré $\mathrm{D}$, et al. Novel dyskerin- mediated mechanism of p53 inactivation through defective mRNA translation. Cancer Res. 2010; 70:4767-4777.

24. Boisvert FM, Hendze, MJ, Bazett-Jones DP. Promyelocytic leukemia (PML) nuclear bodies are protein structures that do not accumulate RNA. J Cell Biol. 2000; 148:283-292.

25. Grandori C, Gomez-Roman N, Felton-Edkins ZA, Ngouenet C, Galloway DA, Eisenman RN, White RJ. c-Myc binds to human ribosomal DNA and stimulates transcription of rRNA genes by RNA polymerase I. Nature Cell Biol. 2005; 7:311-318.

26. Garagnani P, Bacalini MG, Pirazzini C, Gori D, Giuliani C, Mari D, Di Blasio AM, Gentilini D, Vitale G, Collino S, Rezzi S, Castellani G, Capri M, et al. Methylation of ELOVL2 gene as a new epigenetic marker of age. Aging Cell. 2012, 11:1132-1134.

27. Bacalini MG, Pacilli A, Giuliani C, Penzo M, Trerè D, Pirazzini C, Salvioli S, Franceschi C, Montanaro L, Garagnani P. The nucleolar size is associated to the methylation status of ribosomal DNA in breast carcinomas. BMC Cancer. 2014; 14:361.

28. Trerè D. AgNOR staining and quantification. Micron. 2000; 31:127-131. 